\title{
The transverse aortic constriction heart failure animal model: a systematic review and meta-analysis
}

\author{
Lena Bosch ${ }^{1}$ • Judith J. de Haan ${ }^{1}$ - Marissa Bastemeijer ${ }^{1}$ Jennifer van der Burg ${ }^{1}$ • Erik van der Worp ${ }^{1}$. \\ Marian Wesseling ${ }^{1}$ - Margarida Viola ${ }^{1}$. Clémene Odille ${ }^{1} \cdot$ Hamid el Azzouzi $^{1,6}{ }^{1}$ Gerard Pasterkamp ${ }^{2,3}$. \\ Joost P.G. Sluijter ${ }^{1,3} \cdot$ Kimberley E. Wever $^{4} \cdot$ Saskia C.A. de Jager ${ }^{1,5}$
}

Published online: 25 April 2020

(C) The Author(s) 2020

\begin{abstract}
The transverse aortic constriction (TAC) model is frequently used to study adverse cardiac remodeling upon pressure overload. We set out to define the most important characteristics that define the degree of cardiac remodeling in this model. A systematic review and meta-analyses were performed on studies using the TAC mouse/rat model and reporting echocardiographic outcome parameters. We included all animal studies in which a constriction around the transverse aorta and at least one of the predefined echocardiography or MRI outcome parameters were assessed. A total of 502 articles and $>3000$ wild-type, untreated animals undergoing TAC were included in this study and referenced to a control group. The duration of aortic constriction correlated to the degree of adverse remodeling. However, the mouse data is strongly biased by the preferential use of male C57B1/6 mice (66\% of studies). Furthermore, mostly ketamine/xylazine anesthetics, $27 \mathrm{G}$ needle constriction, and silk sutures are used. Nonetheless, despite the homogeneity in experimental design, the model contained a substantial degree of heterogeneity in the functional outcome measures. When looking at study quality, only $12 \%$ reported randomization, $23 \%$ mentioned any sort of blinding, $25 \%$ adequately addressed the outcomes, and an amazingly low percentage (2\%) showed sample size calculation. Meta-analyses did not detect specific study characteristics that explained the heterogeneity in the reported outcome measures, however this might be related to the strong bias towards the use of specific mouse lines, sex as well as age or to poor reporting of characteristics of study quality.
\end{abstract}

Keywords Transverse aortic constriction $\cdot$ Hear failure $\cdot$ Animal model $\cdot$ Systematic review $\cdot$ Meta-analysis

Lena Bosch and Judith J. de Haan contributed equally to this work.

Electronic supplementary material The online version of this article (https://doi.org/10.1007/s10741-020-09960-w) contains supplementary material, which is available to authorized users.

Saskia C.A. de Jager

s.c.a.dejager@umcutrecht.nl

1 Laboratory of Experimental Cardiology, University Medical Center Utrecht, Heidelberglaan 100, 3584 CX Utrecht, Netherlands

2 Central Diagnostics Laboratory, University Medical Center Utrecht, Utrecht, The Netherlands

3 UMC Utrecht Regenerative Medicine Center, Circulatory Health Laboratory, University Utrecht, Utrecht, Netherlands

4 SYstematic Review Centre for Laboratory animal Experimentation (SYRCLE), Department for Health Evidence, Nijmegen Institute for Health Sciences, Radboud University Medical Center, Nijmegen, The Netherlands

5 Laboratory of Translational Immunology, Department of Immunology, University Medical Center Utrecht, Utrecht, The Netherlands

6 Department of Molecular Genetics, Erasmus University Medical Center, Rotterdam, Netherlands

\section{Introduction}

Approximately 23 million people worldwide suffer from heart failure (HF), and this incidence will further increase the coming decades. [1] The development of HF is characterized by a process of adverse cardiac remodeling. [2] To study this process, several animal models are available and a commonly used model is a pressure overload-induced HF by transverse aortic constriction (TAC). First described by Rockman et al in 1991, the TAC model was designed to study the mechanisms behind cardiac hypertrophy. [3] Besides hypertrophy, the model is characterized by cardiac fibrosis, a limited amount of inflammation, and eventually cardiac dilation and HF. [4] Animals subjected to TAC exhibit variable severity of adverse cardiac remodeling [5], and multiple study characteristics can potentially influence the response to TAC. Variability exists in selected animal species, genetic background, sex, follow-up time, and severity of constriction. To which extend these variables affect the degree of cardiac remodeling is not known; however, they are important for interpretation of published 
data and may aid to design optimization of the TAC model. Since the introduction of murine echocardiography, numerous studies using the TAC model were published reporting cardiac dimensions and function as outcome parameters. [6]

Translational failure is common in HF research; therapies with efficacy in preclinical models have failed when applied to human clinical trials. Multiple factors are involved in these disappointing outcomes, including limitations in preclinical animal models, hindering the development of new therapies for HF. [7] Therefore, in this systematic review and metaanalyses, we sought to evaluate the influence of animal characteristics and methodological differences of TAC surgery on echocardiographic outcome parameters. A systematic review of animal studies using the TAC model may provide insight into a number of issues currently impeding translation, such as the characteristics of the animal model and the quality of the published studies. Previously, systematic reviews of preclinical studies have proven useful in design optimization of both preclinical and clinical studies. [8] In this systematic review and meta-analysis, we set out to analyze the influence of variables such as strain, sex, and surgery characteristics on adverse cardiac remodeling after TAC.

\section{Methods}

\section{Search strategy}

A detailed protocol was published online (PROSPERO registration number CRD42017079553; https://www.crd.york.ac. uk/prospero/display_record.php?RecordID=79553) before the start of this systematic review and provided as supplement to this manuscript. [9] This systematic review is based on published results of animal studies that used a transverse aortic constriction (TAC) model in untreated, wild-type animals to induce adverse cardiac remodeling.

\section{Amendments to the review protocol}

Data extraction was performed in duplicate by two individual researchers for 57 (11.4\%) randomly selected included studies, after which the percentage deviation between reviewers was calculated. Since this percentage was very low, $0.8 \%$ for numerical data and $1.2 \%$ for data extracted using digital ruler software, data extraction for the remaining 443 studies was performed by a single reviewer. We chose to leave the secondary outcome analysis because of the results of the primary outcome analysis. Furthermore, we only took mouse for our primary analysis because of limited number of articles for other species and for the understandability of the mean difference instead of standardized mean difference.

\section{Search and study selection}

Pubmed and EMBASE were searched for transverse aortic constriction and its synonyms on 12-04-2017 (see supplemental table $1 \& 2$ for all search terms). A second search was done on 20-03-2019, to update this review. The animal filters developed by SYRCLE were used to select only animal studies. [10] Duplicates were removed in Endnote. We included all animal studies in which TAC surgery was performed, with a constriction around the transverse aorta between the brachiocephalic artery and left common carotid artery, and at least one of the following outcome parameters assessed by echocardiography or MRI were included: end diastolic volume (EDV) or end diastolic diameter (EDD), end systolic volume (ESV) or end systolic diameter (ESD), and/or ejection fraction (EF) or fractional shortening (FS). All animal species and sexes were included. Only studies with a relevant control group (sham surgery, baseline measurements or untreated control animals) were included. Only full publications with original data were included. All studies without TAC or the functional outcome parameters mentioned above were excluded. Animals with comorbidities, genetically modified animals, animals undergoing co-intervention such as compound or solvent administration were excluded. Abdominal, ascending, and descending aortic constriction, angiotensin II infusion, and other ways of inducing hypertension/pressure overload were excluded. Conference abstracts, letters to the editor, and editorials were excluded. Articles not reporting the number of animals were excluded. The systematic review platform SyRF (syrf.org.uk) was used for study selection and extraction of data. Articles were first screened for eligibility based on their title and abstract, and eligible articles were then screened for final inclusion based on full text. Title/abstract screening and full-text screening were performed by at least two independent researchers, and discrepancies were additionally assessed by a third investigator.

\section{Data collection}

From each publication, the following data was extracted: species, genetic background, sex, age, weight, number of animals, TAC duration, characteristics of TAC procedure namely suture material, constriction diameter, anesthetic, follow-up time, and whether a minimally invasive surgery technique was used defined as entering the thorax through the 2 nd intercostal space. When articles reported a range of, for instance, age or weight, the average was taken. Outcome data were extracted for the primary outcomes EDD, EDV, ESD, and ESV and the secondary outcomes FS and EF by echocardiography or MRI, cardiac hypertrophy, and survival. For each outcome, group averages, standard deviation (SD), and number of animals per group $(n)$ were extracted. If the standard error was reported, it was converted to SD. When multiple 
time points were assessed, only data from the latest time point was extracted. When data was presented only graphically, a digital ruler was used for extraction (Universal Desktop Ruler; AVPSOFT).

\section{Risk of bias assessment}

To assess risk of bias, SYRCE's Risk of Bias tool was used [11] with some adaptations. We included reporting of sample size calculations as an additional study quality indicator. In addition, we extracted data on the reporting of any measure of randomization, blinding, or conflict of interest statement. Furthermore, we assessed if outcome data were adequately addressed, defined by reporting the number of animals included in the analysis separately for each outcome measure.

\section{Data synthesis and statistical analysis}

Data were analyzed using STATA/SE, (version 11; StataCorp, College Station, TX, USA). For EDD, EDV ND ESD, and $\mathrm{ESV}$, we calculated the effect size as mean difference (MD) between the TAC group and non-TAC control group (consisting of a mixture of nontreated and sham controls) with corresponding 95\% confidence interval (MD [95\% CI]). To account for anticipated heterogeneity, effect sizes were pooled using random effects meta-analysis, taking into account the precision of individual studies and the variation between studies and weighing each study accordingly. Heterogeneity was quantified using $I^{2}$ and $R^{2}$ statistics. Pre-specified subgroup analyses were performed using meta-regression if at least two subgroups contained $\geq 5$ comparisons. If meta-regression indicated that a subgroup variable explained a significant proportion of between-study heterogeneity, differences between individual strata were determined by performing a $t$ test on the MD or RR and $95 \% \mathrm{CI}$ of these strata.

\section{Results}

\section{Study selection process}

After removal of duplicates, a total of 7742 papers were included, after which 3226 articles were excluded (flow chart in Fig. 1) based on title and abstract. During full-text screening, 3351 articles were further excluded, see Fig. 1 for the number of articles excluded per exclusion criterion. A total of 468 articles were used for data extraction.

\section{Animal characteristics}

The 464 included articles contained a total of 500 comparisons of an experimental group exposed to TAC versus a group measured at baseline or undergoing sham surgery. All included experiments were performed in rodents, of which $95 \%$ (472/500 comparisons) in mice and 5\% (28/500) in rats. Data were predominantly obtained from male animals $(66 \%$, $332 / 500)$. Females were used in 5\% (21/500) of comparisons, $8 \%(45 / 500)$ used a mixed group of animals, and 21\% (102/ 500) did not report the sex of the animals used (Fig. 2a). Various different mouse strains were used (Fig. 2b). Most commonly C57BL/6 mice $(67 \%, 344 / 472)$, for $10 \%(46 /$ 472) of the comparisons, the mouse strain was not specified. Median age of the mice was 10 weeks, ranging from 3 to 56 weeks, and 7 weeks for rats (range: 3-12 weeks). The median body weight was $25 \mathrm{~g}$ for mice (range: $20-36 \mathrm{~g}$ ) and $155 \mathrm{~g}$ for rats (range: $45-250 \mathrm{~g}$ ).

\section{Surgery characteristics}

As the vast majority of studies was performed in mice and in order to improve clarity and readability of the manuscript, we decided to continue further on sub analysis in mouse studies only. For TAC or sham surgery, mainly ketamine and xylazine $(\approx 40 \%, 191 / 500)$ or isoflurane $(\approx 30 \%, 171 / 500)$ were used as anesthetic (Fig. 3a). Thirty-five percent of the experiments were performed using minimally invasive techniques, $32 \%$ was not performed minimally invasively, and $33 \%$ did not specify this (respectively 174,162 , and 164 out of 500 comparisons). The most frequently used technique to apply TAC was placing a suture around the transverse aorta $(80 \%, 400 /$ 500). Of the studies reporting suture type, 53\% (204/383 comparisons) used a silk suture (Fig. 3b), while 25\% (95/383) made use of polyamide and $12 \%$ (47/383) of a prolene suture. In $4 \%(20 / 500)$ of the comparisons, a clip was used to apply TAC, and $16 \%(80 / 500)$ did not mention the material or method used for TAC induction. The TAC diameter was reported in $373 / 500$ comparisons in gauge $(G)$ of the needle used, which ranged from 17 to $30 \mathrm{G}$. In most cases, a $27 \mathrm{G}$ needle was used $(71 \% ; 265 / 373)$, followed by $26 \mathrm{G}(12 \% ; 45 / 373)$ and $25 \mathrm{G}$ $(7 \% ; 26 / 373)$. In $33 \%$ of cases $(154 / 500)$, confirmation of TAC after surgery was reported, which was performed by echo/doppler in 122 of these comparisons and in 42 by invasive pressure measurements. In 66\% (336/500) of the studies, either confirmation of pressure overload on the heart was not assessed or it was not reported (Fig. 3c). After surgery, followup time ranged from 3 to 280 days. Most experiments used a follow-up time of 28 days $(21 \% ; 107 / 500)$, followed by 14 and 56 days (both $10 \% ; 53 / 500)$ and 7 days $(6 \% ; 32 / 500)$. For functional outcome assessment, most comparisons (96\%; 387/ $500)$ used $2 \mathrm{D}$ echocardiography. In a small proportion of the studies (3\%, 13/500 comparisons), MRI was used.

\section{Study quality assessment}

The overall reporting of study quality indicators is presented in Fig. 4. Out of the total of 464 included studies, 342 (74\%) 
Fig. 1 Flow chart of study selection process. Our systematic search in Pubmed and EMBASE yielded 7742 unique publications. After title and abstract screening, articles were screened full text of which 4355 were excluded based on exclusion criteria. Data from 464 articles was included in metaanalysis and quality assessment. TAC transverse aortic constriction, admin administration, other possible reasons: additional surgery such as ovariectomy, methods not mentioned clearly, for instance duration of TAC not stated, NR not reported

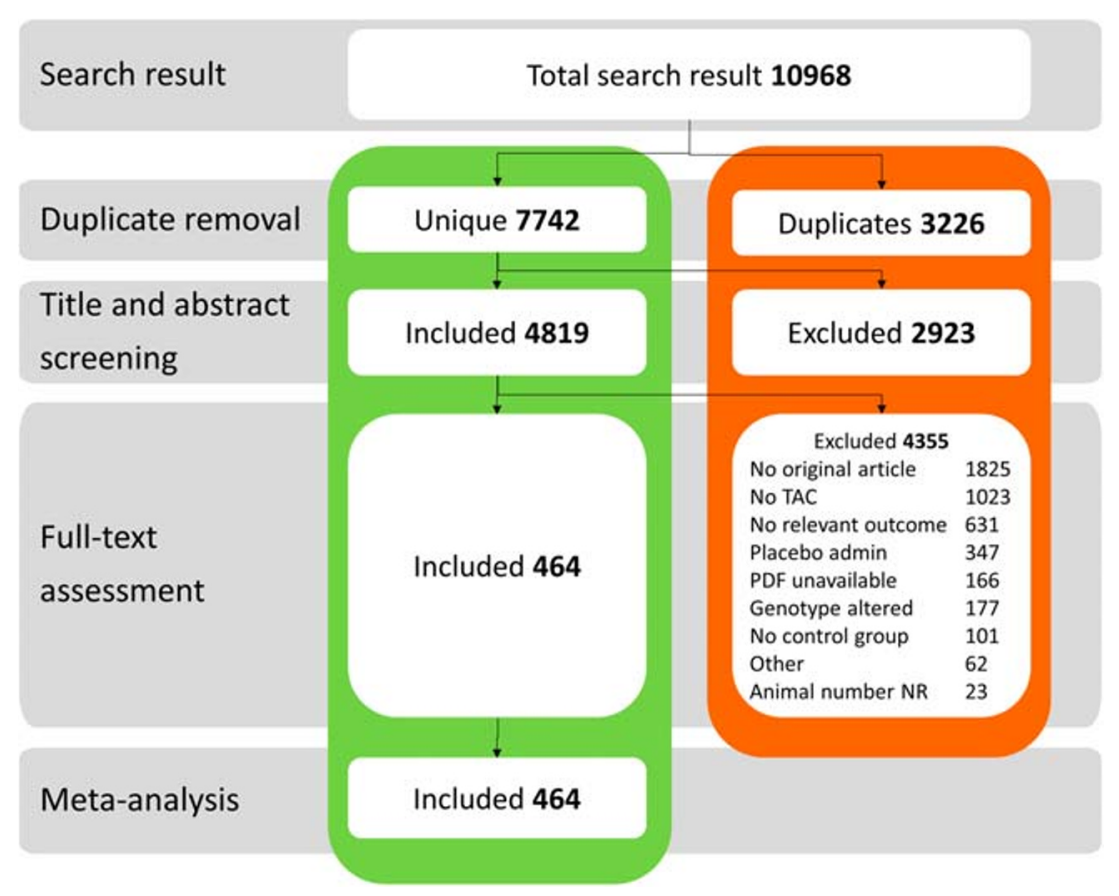

included a conflict of interest statement, of which the vast majority $(325,95 \%)$ stated to have no conflict of interest. Only $23 \%(n=108)$ studies reported the number of animals included in the analysis separately for each outcome measure (depicted as outcome data adequately addressed). Therefore, it cannot be ruled out that the majority of studies have excluded a number of animals for some of the outcome assessments. Unfortunately, the (predefined) criteria for exclusion were not reported. A limited number of studies reported randomization of animals (12\%) or blinding $(23 \%)$ of the investigators during the study at any level (allocation of the animals, blinding of researchers performing the experiment, or blinding during follow-up analysis). Only 11 studies (2\%) reported a sample size calculation. Taken together, these data implicate a substantial risk of bias in the majority of published articles using the TAC model.

\section{Meta-analysis}

Despite the substantial risk of bias and the preference for using male $\mathrm{C} 57 \mathrm{Bl} / 6$ mice, we investigate what the differences are in response to pressure overload between the sexes and different mouse strains. With these data in hand, we aim to aid to validated decision making regarding the choice for a specific animal model, strain, and sex. TAC induces severe structural remodeling of the heart, and we defined end diastolic (end diastolic diameter (EDD) and volume (EDV)) and end systolic diameter (ESD) and volume (ESV)) function as primary outcome measures. In a total of 341 comparisons with 3370 mice subjected to TAC, we observed an increase in EDD of $0.35 \mathrm{~mm}$ [0.3$0.4 ; I^{2} 91.2 \% p<0.0001$, Fig. 5a] compared with 2984 control mice. Total 398 mice subjected to TAC displayed

\section{a Sex}

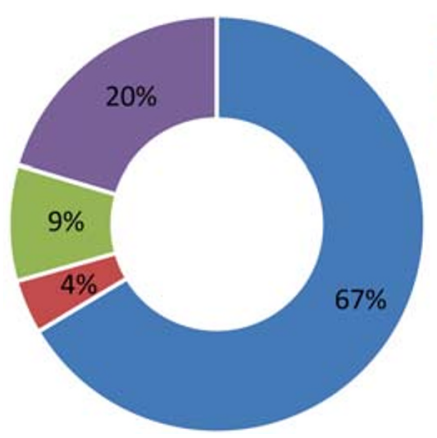

\section{b Mouse strains}

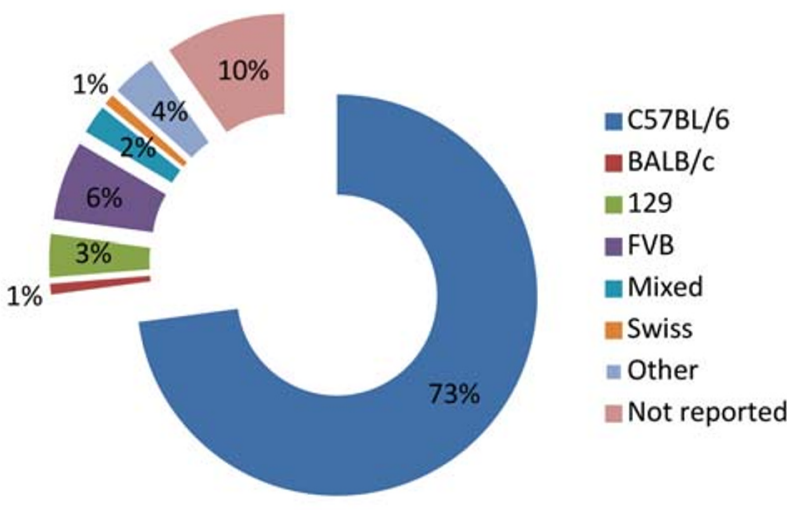

Fig. 2 Distribution of sex and mouse strain. Distribution of a sex of the animals in all included studies and $\mathbf{b}$ mouse strains of included mouse studies (absolute numbers of a total of 500) 


\section{a Anesthesia}

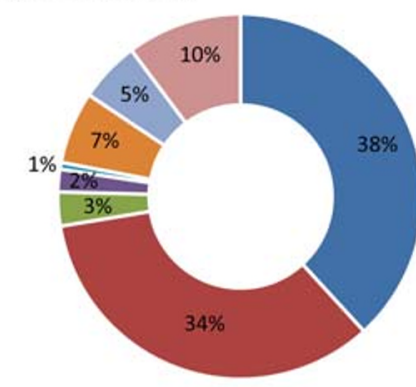

C Degree of constriction

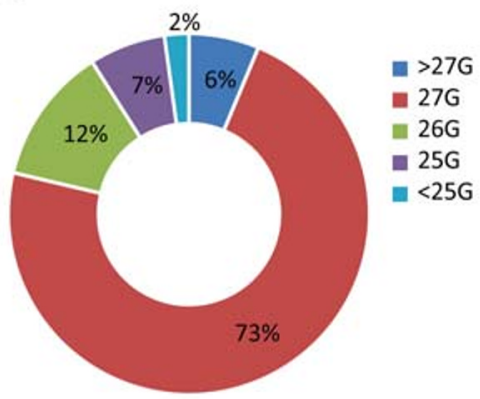

e Method of constriction and suture material

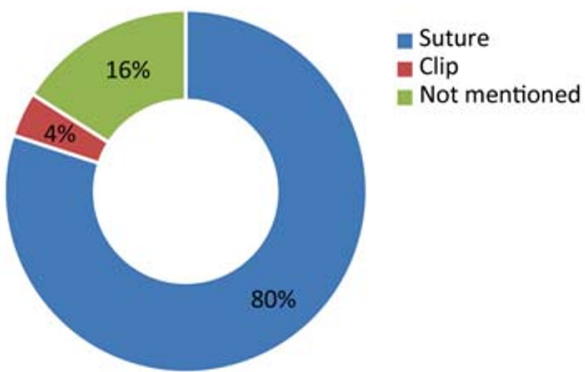

\section{b Surgical Technique}

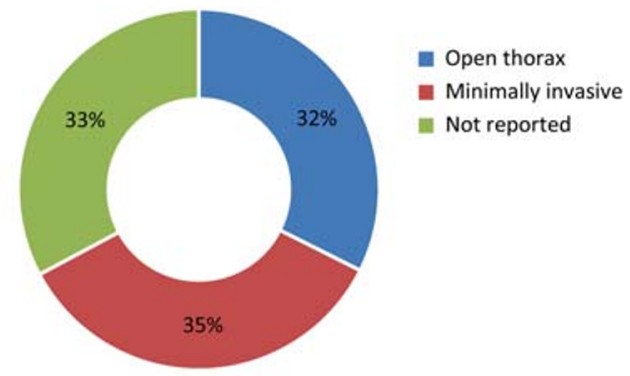

d Confirmation of pressure overload

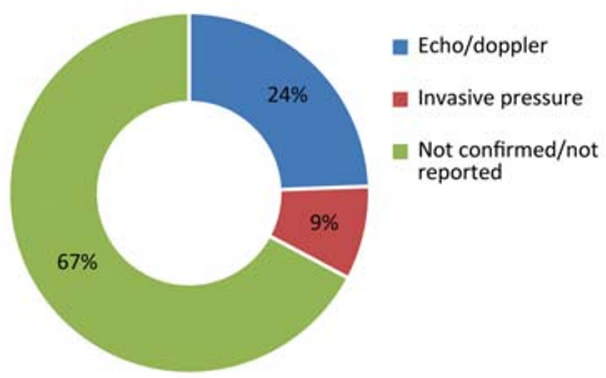

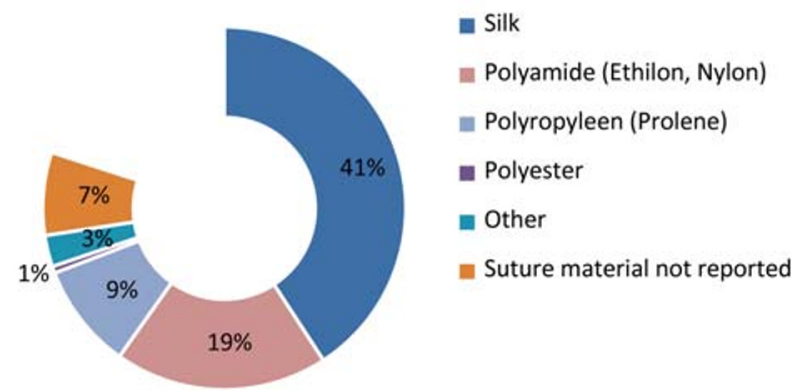

Fig. 3 Study characteristics. Distribution of a anesthetics used during TAC or sham surgery, b surgical technique, $\mathbf{c}$ degree of constriction, $\mathbf{d}$ confirmation of pressure overload, and e method of constriction and suture materials (absolute numbers of a total of 500)

an average EDV increase of $20.09 \mu 1$ [15.7-24.4; $I^{2} 92.8 \%$ $p<0.0001$, Fig. 5b] compared with 356 control mice from a total of 39 studies/comparisons. When assessing systolic function, ESD increased with $0.50 \mathrm{~mm}\left[0.43-0.58 ; I^{2}\right.$ $16.0 \% p=0.017$, Fig. $5 \mathrm{c}]$ in 2615 mice subjected to TAC referenced to 2383 control mice derived from 270 studies. Based on 35 studies ESV was on average increased by $28.74 \mu \mathrm{l}$ [20.8-36.68; $I^{2} 99.9 \% p<0.0001$, Fig. 5d] based on 345 mice subjected to TAC compared with 299 control mice.
Fig. 4 Data quality. Reporting of study quality indicators; conflict of interest statement, outcome data adequately addressed, blinding, randomization, and sample size calculation in the 464 included articles

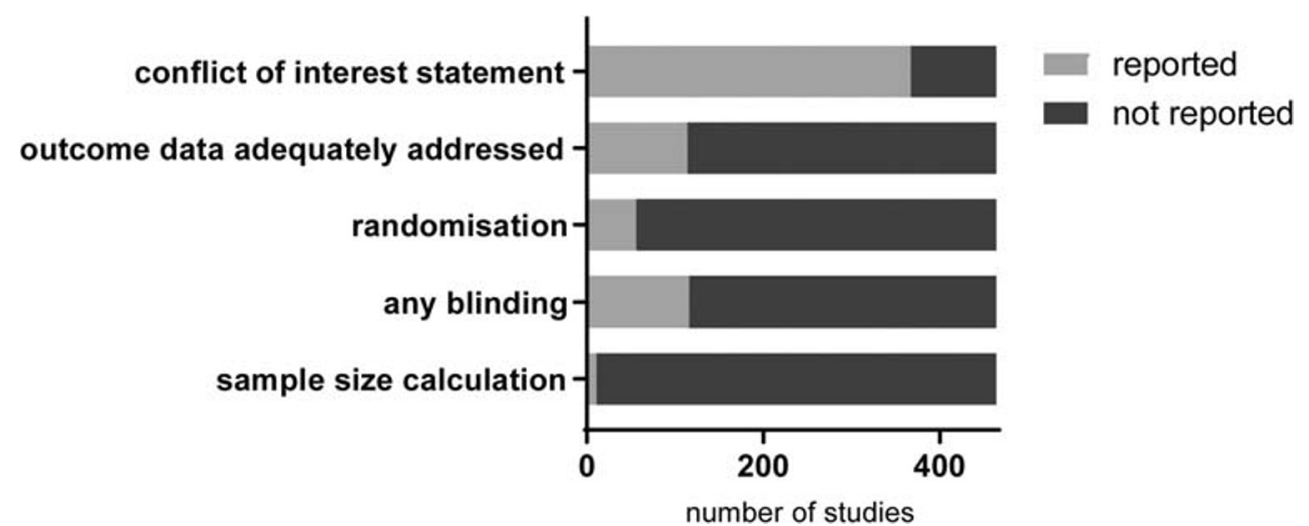




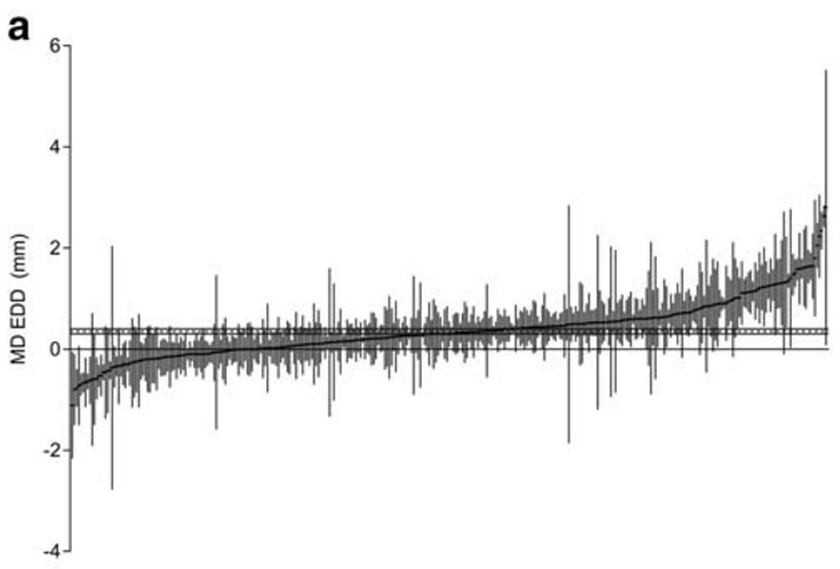

C

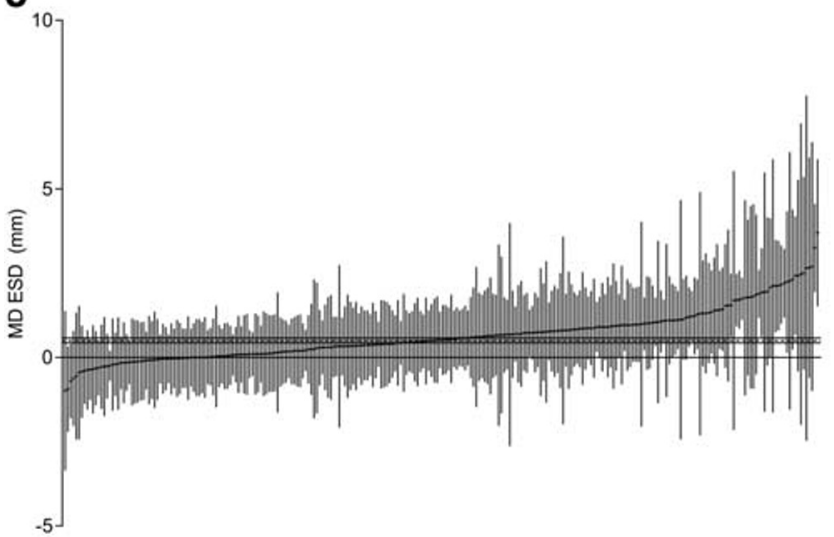

Fig. 5 TAC induces severe structural remodeling of the heart. Forest plots of the effect of TAC on a end diastolic diameter (EDD; $n=314$ comparisons), b end diastolic volume (EDV; $n=39$ ), $\mathbf{c}$ end systolic diameter (ESD; $n=270)$, and $\mathbf{d}$ end systolic volume (ESV; $n=35$ ).

\section{Subgroup analysis}

Subgroup analyses were performed to identify sources of between-study heterogeneity. Table 1 summarizes the influence of the following study characteristics on the level of heterogeneity: sex, strain, and confirmation of the TAC. None of the investigated variables explained a significant proportion of the heterogeneity present in the overall analysis. Due to low number of comparisons for ESV/EDV, we did not investigated heterogeneity in these outcome parameters.

\section{Influence of sex and strain on TAC model}

TAC surgery was effective in both sexes and did not differ depending on the sex of the animals (male versus female versus mixed versus not known) for any of the outcome measures (Fig. 6 a and $b$ show the influence of sex on EDD and ESD, respectively). The strain of the mouse also did not account for a significant proportion of the heterogeneity in the overall analysis. The major differences are observed between $\mathrm{C} 57 \mathrm{Bl} / 6$ and $\mathrm{Balb} / \mathrm{C}$ mice; however, it must be noted that

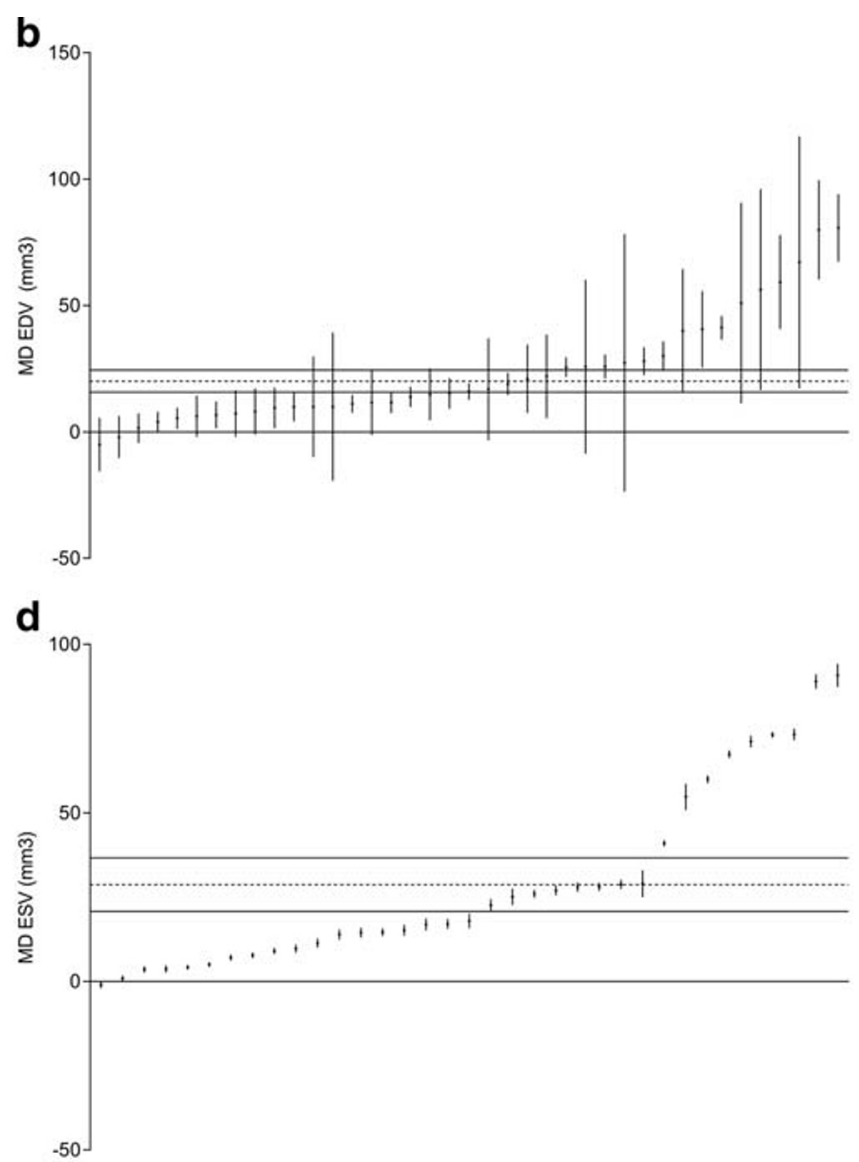

Data are presented as raw mean difference (MD) and 95\% confidence intervals (95\% CI). In all analyses, the pooled MD and 95\% CI (horizontal dotted line and black line) indicate an increase outcome after TAC, when compared with controls

these outcomes may be biased as a consequence of the large proportion of studies that used C57BL/6, while only very limited numbers used Balb/c mice. In Fig. $6 \mathrm{c}$ and $d$, the effect of mouse strain on EDD and ESD (MD) is shown. Also confirmation of proper placement of the TAC and thus cardiac pressure overload did not affect EDD and ESD (Fig. 6e, f).

\section{Influence of duration and diameter of constriction on TAC severity}

The duration of constriction varied from 3 to 280 days after TAC placement. Linear regression showed borderline significance for the effect of TAC duration on the primary outcome $\operatorname{EDD}(p=0.061$, Fig. 7a), while a stronger effect of constriction duration on the ESD was observed ( $p=0.001$, Fig. $7 b$ ). The constriction diameter of the needle used to apply the TAC varied from 17 to $30 \mathrm{G}$. However, most data clustered around $27 \mathrm{G}$. Linear regression did not show a significant relation between constriction diameter and $\operatorname{EDD}(p=0.117)$ or ESD ( $p=0.088$, data not shown). 
Table 1 Influence of: sex, strain, and confirmation of the TAC on the level of heterogeneity in EDD and ESD

\begin{tabular}{|c|c|c|c|c|c|c|}
\hline & \multicolumn{3}{|c|}{ EDD } & \multicolumn{3}{|c|}{ ESD } \\
\hline & $\#$ & $\mathrm{MD}(\mathrm{mm})$ & {$[95 \% \mathrm{CI}]$} & $\#$ & $\mathrm{MD}(\mathrm{mm})$ & {$[95 \% \mathrm{CI}]$} \\
\hline Sex & \multicolumn{3}{|c|}{$p=0.41$, adj. $R^{2}=-0.44 \%$} & \multicolumn{3}{|c|}{$p=0.85$ adj. $R^{2}=-3.38 \%$} \\
\hline Female & 13 & 0.26 & $-0.02,0.55$ & 8 & 0.58 & $0.13,1.04$ \\
\hline Male & 217 & 0.38 & $0.31,0.45$ & 179 & 0.53 & $0.43,0.62$ \\
\hline Mixed & 36 & 0.35 & $0.18,0.53$ & 24 & 0.48 & $0.24,0.73$ \\
\hline Not reported & 75 & 0.27 & $0.15,0.39$ & 59 & 0.45 & $0.29,0.61$ \\
\hline Mouse strain & \multicolumn{3}{|c|}{$p=0.04$, adj. $R^{2}=2.84 \%$} & \multicolumn{3}{|c|}{$p=0.11$ adj. $R^{2}=5.51 \%$} \\
\hline 129 & 15 & 0.14 & $-0.12,0.39$ & 14 & 0.45 & $0.29,0.61$ \\
\hline $\mathrm{Balb} / \mathrm{c}$ & 2 & -0.23 & $-1.05,0.60$ & 3 & 0.53 & $0.43,0.62$ \\
\hline $\mathrm{C} 57 \mathrm{BL} / 6$ & 250 & 0.40 & $0.34,0.47$ & 194 & 0.53 & $0.43,0.62$ \\
\hline FVB & 22 & 0.11 & $-0.11,0.32$ & 15 & 0.58 & $0.13,1.04$ \\
\hline Mixed & 7 & 0.10 & $-0.33,0.52$ & 6 & 0.53 & $0.43,0.62$ \\
\hline Other & 12 & 0.21 & $-0.08,0.50$ & 12 & 0.53 & $0.43,0.62$ \\
\hline Swiss & 3 & -0.03 & $-0.61,0.55$ & 3 & 0.45 & $0.29,0.61$ \\
\hline Not reported & 30 & 0.21 & $0.20,0.58$ & 23 & 0.45 & $0.29,0.61$ \\
\hline TAC confirmed & \multicolumn{3}{|c|}{$p=0.63$, adj. $R^{2}=-0.36 \%$} & \multicolumn{3}{|c|}{$p=0.66$ adj. $R^{2}=-1.52 \%$} \\
\hline Echo/doppler & 85 & 0.34 & $0.22,0.45$ & 63 & 0.51 & $0.35,0.68$ \\
\hline Invasive pressure & 33 & 0.27 & $0.10,0.45$ & 29 & 0.40 & $0.17,0.64$ \\
\hline Not reported & 223 & 0.37 & $0.30,0.43$ & 178 & 0.52 & $0.42,0.62$ \\
\hline
\end{tabular}

\section{Discussion}

This systematic review and meta-analyses provide an overview of the commonly used animal model to study adverse cardiac remodeling: transverse aortic constriction (TAC). As expected, TAC significantly increased the EDD, EDV, ESD, and ESV indicative of adverse cardiac remodeling and heart failure.

\section{Animal characteristics}

A clear preference for certain animal characteristics was seen. First, only rodents (mice and rats) were included in our study. During the screening phase, we noticed that in large animals (such as pigs and dogs), only a constriction of the ascending or descending aorta is used. Therefore, these animal species were not included in our review. Second, most of the articles used male animals, a very little proportion of studies was performed in female animals only. A troublesome $21 \%$ failed to report the sex of the animals. This sex bias, which is widespread in clinical and preclinical studies, is reason for concern. [12] Previously, a systematic review showed that the sex of the animals used was not reported in $20 \%$ of published cardiovascular animal research. Males were exclusively used in the majority $(72 \%)$ of studies, whereas only $13 \%$ of articles reported the use of female animals. [13] These numbers are similar to our findings in for TAC model. In subgroup analysis, we were not able to show any effects of sex on the outcome parameters. Clinical studies document that female patients with similar degree of aortic stenosis more frequently demonstrate thick-walled chambers, corresponding with concentric left ventricular hypertrophy, compared with male patients. [14, 15] However, in preclinical studies, the distinguishing between concentric and eccentric hypertrophy is rarely made. Regarding mouse strain, a clear preference is seen for C57BL/6, used in $73 \%$ of the included mouse studies. A previous study showed that even the specific substrains of C57BL/6 differ in their (hypertrophic) response to TAC, indicating that genetic background might be of influence on the cardiac outcome after TAC. [16] However, in our subgroup analysis, no statistical significant proportion of between-study heterogeneity could be attributed to mouse strain for any outcome measure. However, this may be due to limited data available for certain strains or a lack of reporting on the specific C57BL/6 substrain used.

\section{Surgery characteristics}

The standard surgery procedure in TAC models is to perform a partial thoracotomy for visualization of the aortic arch. This open-chest model leads to changes in respiratory physiology caused by the thoracotomy. To prevent this, a minimally invasive surgical technique through the 2 nd intercostal space was developed, leading to a more rapid recovery of the mice. [17] 
Fig. 6 Influence of sex, mouse strain, and confirmation of pressure overload on end diastolic and end systolic diameters. Effect of sex (a), mouse strain (b), and confirmation of pressure overload on the outcome parameters EDD and ESD (MD). Horizontal dotted and solid lines represent pooled effect and its $95 \%$ confidence interval. In each bar, the number of comparisons contributing data is indicated a

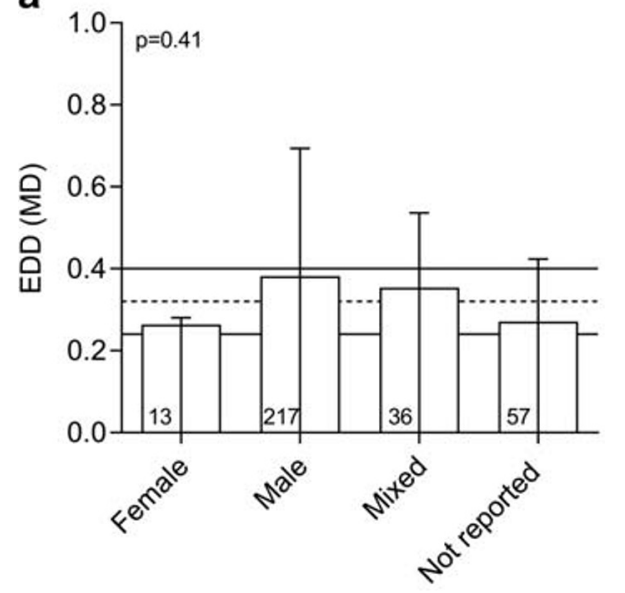

C

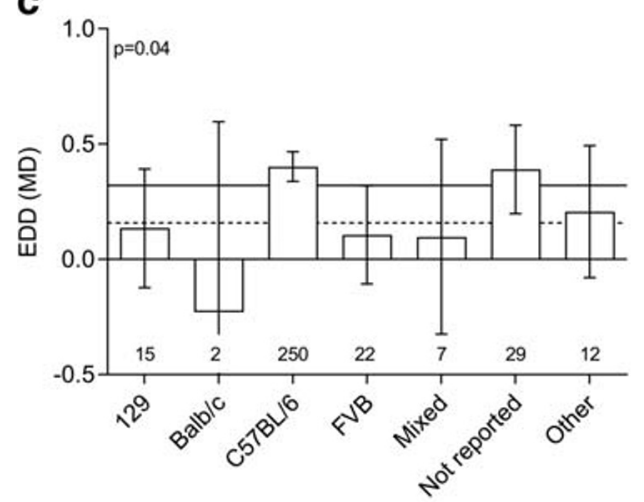

e
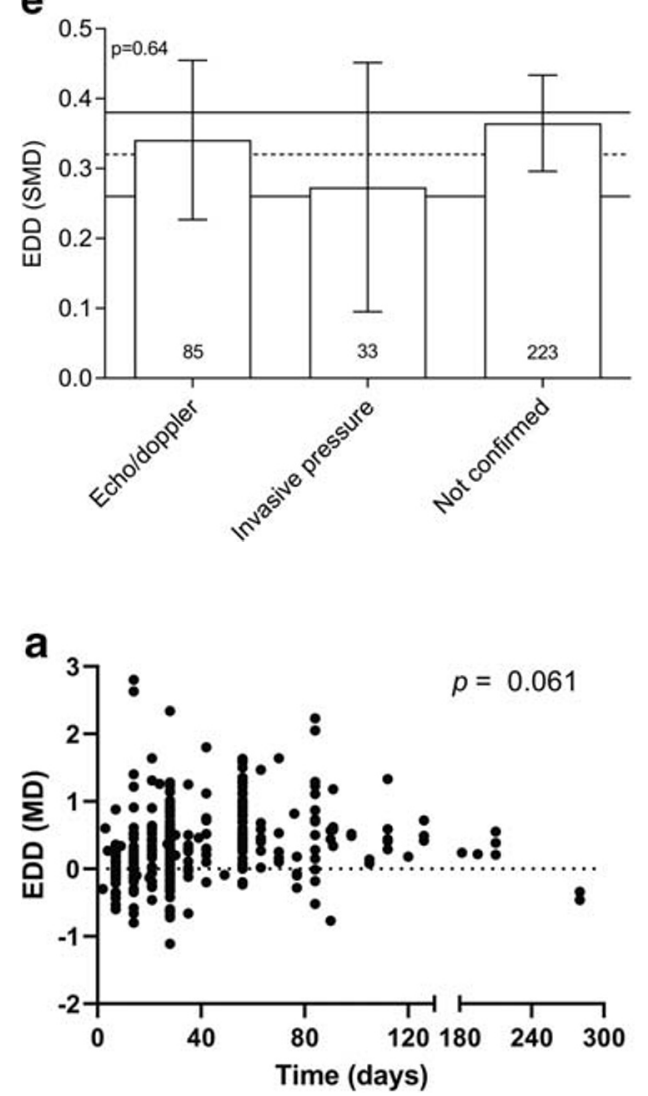

b

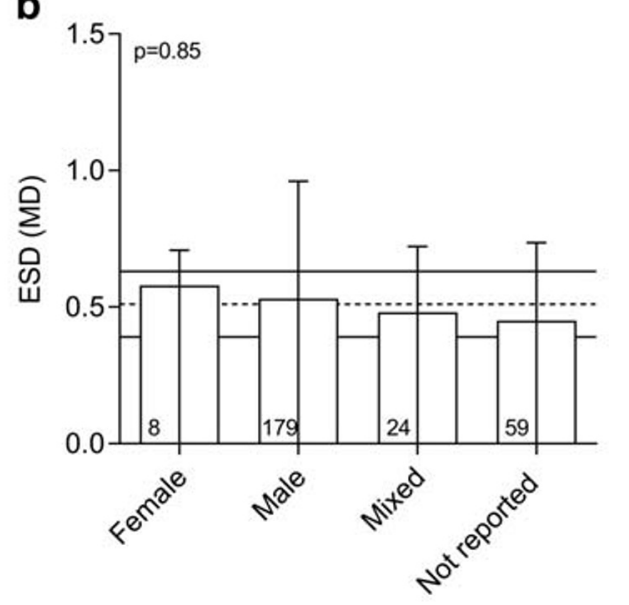

d

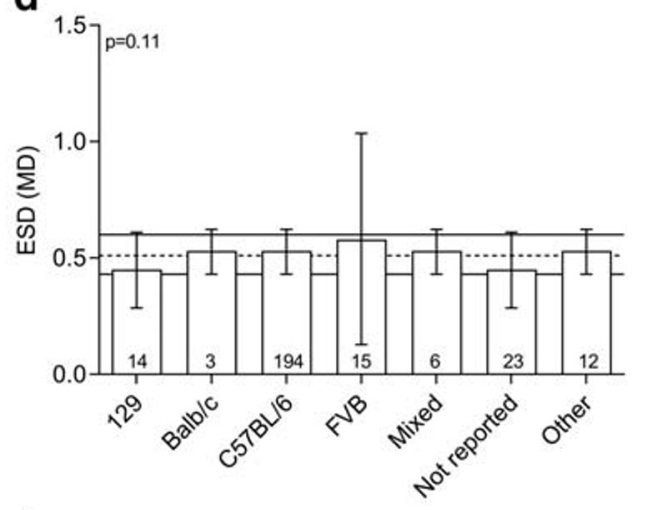

f

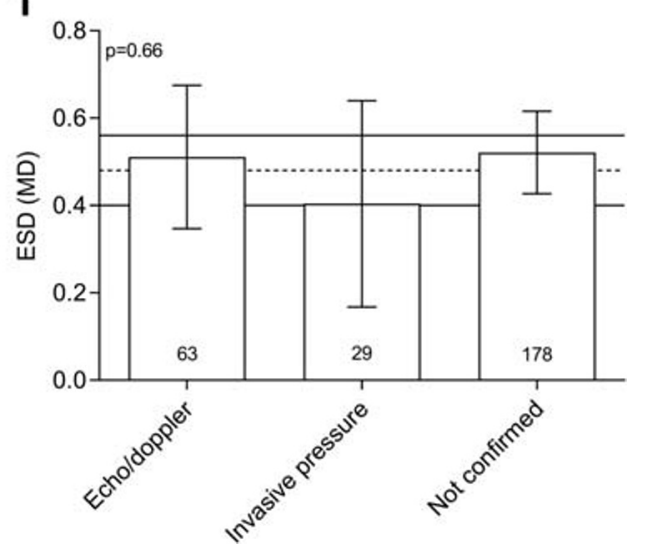

b

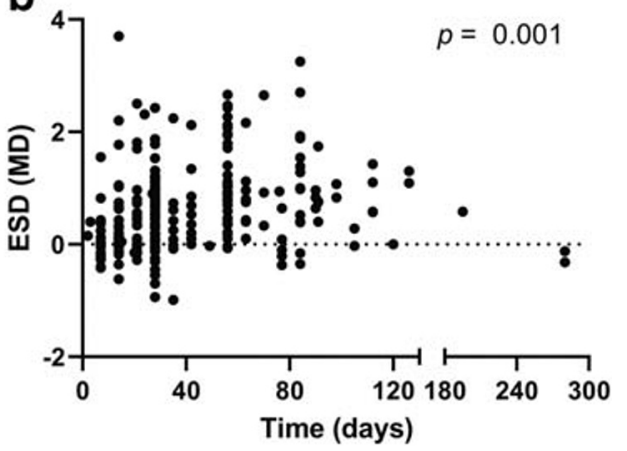


Minimal invasive surgery was performed in $35 \%$ of the included studies. After surgery, $34 \%$ of studies mentioned confirmation of the TAC by either echo or invasive pressure measurements. This means that a relatively large proportion of studies may not have confirmed the constriction. Doppler echocardiography is a relatively easy and noninvasive technique to assess TAC severity without compromising future vascular access. [5] From our own experience, we know that sometimes a suture loosens, preventing adverse cardiac remodeling from happening. The low percentage of TAC confirmation might explain why a proportion of studies showed no or only very little adverse remodeling.

The majority of studies ( $71 \%$ ) made use of $27 \mathrm{G}$ needles for TAC placement. No association between needle size and the outcome parameters was however observed. This is possibly due to clustering of data points around $27 \mathrm{G}$ since relatively large or small needles were less frequently used. Interestingly, only a marginal significant effect of TAC duration on one outcome parameter (EDD) was present. This was unexpected since a longer duration of TAC would generally lead to increased adverse remodeling and changes in all outcomes. Absence of association there might point towards inconsistency of the model causing large variability between animals and studies.

\section{Effect of TAC on the outcome parameters}

When compared with control animals, TAC results in an increase in cardiac diameters and volumes (EDD, EDV, ESD, and ESV). Mice subjected to TAC exhibit variable severity of adverse remodeling. It was previously shown that independent of study characteristics, such as sex, strain, needle size, or time course, considerable variability exists within the model even when performed at the same laboratory. [5] Part of the animals show compensated LV hypertrophy, while only a subset of animals develop overt HF. Understanding this variability involved in the model is crucial to appropriate study design and interpretation, particularly when effects of conditional expression of genetic interventions or pharmacologic agents are studied. Variability within the model will affect the sample size required to detect the effect of treatment or genetic alterations. Of the included studies, $16-17 \%$ used $\leq 5$ animals in the TAC group, and since only 7 out of 371 studies reported a power calculation, reproducibility of these results might be of concern [5]. The original Rockman technique [3] induces an identical flow area for each animal, regardless of its habitus, consequently causing a variable degree of aortic constriction. This leads to a greater pressure overload on the LV in heavier animals compared with smaller ones. Adjusting the constriction to the size of the mouse and the aorta can lead to more homogeneity of the results. [18] In our sub-analysis, we did not observe a significant influence of the average weight in each study. However, individual differences between mice per study are likely to exist.

\section{Study quality}

Seventy-four percent of included papers mentioned a conflict of interest statement, of which most reported no conflicts of interest. Unfortunately, the other study quality indicators were very poorly reported. Only $23 \%$ reported blinding of the investigator during the study at any level, and only 10 studies reported a sample size calculation. Most articles did not report the number of animals used for each outcome measure. Adequate reporting of these methodological details is crucial to determine the risk of bias and to assess the quality of the evidence. [19] When the TAC model is used to study effects of treatment on adverse remodeling, underreporting of research methodology is often associated with an overestimation of effects. [20] As previously mentioned, we noticed that the number of animals in some studies was very low, and this might contribute to translational failure of preclinical studies. Since most of the studies did not include a power calculation, we cannot exclude the possible effect of under powering on our meta-analysis.

\section{Limitations of this review}

Twenty-three studies were excluded because no animal numbers were reported. Furthermore, we had to exclude various articles because we could not confirm whether TAC was performed or another type of aortic banding. Both reasons indicate also low quality of methodological reporting and increase the risk of bias. However, because we excluded these papers, we cannot state this with certainty. In our search, we used for our control group both sham-operated animals as not-operated animals. This could have caused variation in our data, which we did not take into account. Data extraction was mainly performed by one reviewer, but because of the small between-reviewer deviations observed in the random sample, the effect is likely to be small. A full risk of bias assessment was not yet performed, and the very high amount of heterogeneity remains unexplained.

\section{Conclusion}

The general TAC study is performed using a similar set-up as originally described by Rockman et al. [3], using male C57BL/6 mice of 10 weeks old with ketamine and xylazine as anesthetic, tightening the constriction around a $27 \mathrm{G}$ needle using a silk suture. Follow-up time is generally 28 days, and echocardiographic outcome parameters are assessed using 2D echocardiography. The murine TAC model is a very valuable but also variable animal model. For the future, especially 
when the TAC model is used to test pharmacological interventions, it is imperative that adequate numbers of mice are used, both sexes are studied, and the severity of constriction is confirmed after TAC or new operation techniques are applied to limit variability within the model.

Funding information ZonMw provided financial support for this study with the "De praktijk van een 'Synthesis of Evidence' voor dierexperimenteelonderzoek” grant (project number 114024108).

\section{Compliance with ethical standards}

Conflict of interest The authors declare that they have no conflict of interest.

Open Access This article is licensed under a Creative Commons Attribution 4.0 International License, which permits use, sharing, adaptation, distribution and reproduction in any medium or format, as long as you give appropriate credit to the original author(s) and the source, provide a link to the Creative Commons licence, and indicate if changes were made. The images or other third party material in this article are included in the article's Creative Commons licence, unless indicated otherwise in a credit line to the material. If material is not included in the article's Creative Commons licence and your intended use is not permitted by statutory regulation or exceeds the permitted use, you will need to obtain permission directly from the copyright holder. To view a copy of this licence, visit http://creativecommons.org/licenses/by/4.0/.

\section{References}

1. Bui AL, Horwich TB, Fonarow GC (2011) Epidemiology and risk profile of heart failure. Nat Rev Cardiol 8(1):30-41. https://doi.org/ 10.1038/nrcardio.2010.165

2. Frantz S, Bauersachs J, Ertl G (2009) Post-infarct remodelling: contribution of wound healing and inflammation. Cardiovasc Res 81(3):474-481. https://doi.org/10.1093/cvr/cvn292

3. Rockman HA, Ross RS, Harris AN, Knowlton KU, Steinhelper ME, Field LJ, Ross J Jr, Chien KR (1991) Segregation of atrialspecific and inducible expression of an atrial natriuretic factor transgene in an in vivo murine model of cardiac hypertrophy. Proc Natl Acad Sci U S A 88(18):8277-8281

4. Xia Y, Lee K, Li N, Corbett D, Mendoza L, Frangogiannis NG (2009) Characterization of the inflammatory and fibrotic response in a mouse model of cardiac pressure overload. Histochem Cell Biol 131(4):471-481. https://doi.org/10.1007/s00418-008-0541-5

5. Mohammed SF, Storlie JR, Oehler EA, Bowen LA, Korinek J, Lam CS, Simari RD, Burnett JC Jr, Redfield MM (2012) Variable phenotype in murine transverse aortic constriction. Cardiovasc Pathol 21(3):188-198. https://doi.org/10.1016/j.carpath.2011.05.002

6. Hoit BD, Walsh RA (1997) In vivo echocardiographic assessment of left ventricular function in transgenic and gene-targeted mice. Trends Cardiovasc Med 7(4):129-134. https://doi.org/10.1016/ s1050-1738(97)00003-0

7. Lara-Pezzi E, Menasche P, Trouvin JH, Badimon L, Ioannidis JP, Wu JC, Hill JA, Koch WJ, De Felice AF, de Waele P, Steenwinckel V, Hajjar RJ, Zeiher AM (2015) Guidelines for translational research in heart failure. J Cardiovasc Transl Res 8(1):3-22. https:// doi.org/10.1007/s12265-015-9606-8
8. Pound P, Ebrahim S, Sandercock P, Bracken MB, Roberts I (2004) Where is the evidence that animal research benefits humans? Bmj 328(7438):514-517. https://doi.org/10.1136/bmj.328.7438.514

9. de Haan J BL, van der Burg J, Wever K, el Azzouzi H, de Jager S, Pasterkamp G (2017) Animal models of adverse cardiac remodeling after transverse aortic constriction: the influence of species, strain and sex. PROSPERO: international prospective register of systematic reviews CRD42017079553

10. Hooijmans CR, Tillema A, Leenaars M, Ritskes-Hoitinga M (2010) Enhancing search efficiency by means of a search filter for finding all studies on animal experimentation in PubMed. Lab Anim 44(3): 170-175. https://doi.org/10.1258/la.2010.009117

11. Hooijmans CR, Rovers MM, de Vries RB, Leenaars M, RitskesHoitinga M, Langendam MW (2014) SYRCLE's risk of bias tool for animal studies. BMC Med Res Methodol 14:43. https://doi.org/ 10.1186/1471-2288-14-43

12. Gohar A, Schnabel RB, Hughes M, Zeller T, Blankenberg S, Pasterkamp G, den Ruijter H (2016) Underrepresentation of sex in reporting traditional and emerging biomarkers for primary prevention of cardiovascular disease: a systematic review. Eur Heart J Qual Care Clin Outcomes 2(2):99-107. https://doi.org/10.1093/ ehjqcco/qcv028

13. Ramirez FD, Motazedian P, Jung RG, Di Santo P, MacDonald Z, Simard T, Clancy AA, Russo JJ, Welch V, Wells GA, Hibbert B (2017) Sex bias is increasingly prevalent in preclinical cardiovascular research: implications for translational medicine and health equity for women: a systematic assessment of leading cardiovascular journals over a 10-year period. Circulation 135(6):625-626. https://doi.org/10.1161/circulationaha.116.026668

14. Petrov G, Regitz-Zagrosek V, Lehmkuhl E, Krabatsch T, Dunkel A, Dandel M, Dworatzek E, Mahmoodzadeh S, Schubert C, Becher E, Hampl H, Hetzer R (2010) Regression of myocardial hypertrophy after aortic valve replacement: faster in women? Circulation 122(11 Suppl):S23-S28. https://doi.org/10.1161/circulationaha.109. 927764

15. Carroll JD, Carroll EP, Feldman T, Ward DM, Lang RM, McGaughey D, Karp RB (1992) Sex-associated differences in left ventricular function in aortic stenosis of the elderly. Circulation 86(4):1099-1107

16. Garcia-Menendez L, Karamanlidis G, Kolwicz S, Tian R (2013) Substrain specific response to cardiac pressure overload in C57BL/6 mice. Am J Physiol Heart Circ Physiol 305(3):H397H402. https://doi.org/10.1152/ajpheart.00088.2013

17. Eichhorn L, Weisheit CK, Gestrich C, Peukert K, Duerr GD, Ayub MA, Erdfelder F, Stockigt F (2018) A closed-chest model to induce transverse aortic constriction in mice. J Visual Exp : JoVE 134. https://doi.org/10.3791/57397

18. Merino D, Gil A, Gomez J, Ruiz L, Llano M, Garcia R, Hurle MA, Nistal JF (2018) Experimental modelling of cardiac pressure overload hypertrophy: modified technique for precise, reproducible, safe and easy aortic arch banding-debanding in mice. Sci Rep 8(1):3167. https://doi.org/10.1038/s41598-018-21548-x

19. Kilkenny C, Browne WJ, Cuthill IC, Emerson M, Altman DG (2010) Improving bioscience research reporting: the ARRIVE guidelines for reporting animal research. PLoS Biol 8(6): e1000412. https://doi.org/10.1371/journal.pbio.1000412

20. Jonker SJ, Menting TP, Warle MC, Ritskes-Hoitinga M, Wever KE (2016) Preclinical evidence for the efficacy of ischemic postconditioning against renal ischemia-reperfusion injury, a systematic review and meta-analysis. PLoS One 11(3):e0150863. https://doi.org/10.1371/journal.pone.0150863

Publisher's note Springer Nature remains neutral with regard to jurisdictional claims in published maps and institutional affiliations. 